\title{
N-terminal Pro-Brain Natriuretic Peptide, Adjuvant Laboratory Marker of Acute Phase of Kawasaki Disease in Emergency Department
}

Hyuksøol Kwøn, M.D.

\section{N-terminal Pro-Brain Natriuretic Peptide, Adjuvant Laboratory Marker of Acute Phase of Kawasaki Disease in Emergency Department}

Hyuksool Kwon, M.D.

Department of Emergency Medicine, Seoul National University Bundang Hospital

Purpose: Diagnosis of Kawasaki disease (KD) in emergency department (ED) is sometimes difficult because of unsatisfied diagnostic criteria, especially when febrile days are less than 5 days. Clinical and laboratory features may be helpful in establishing the diagnosis in these cases. N-terminal pro-brain natriuretic peptide (NT-proBNP) has been proved as a laboratory indicator for identifying patients with KD, but usefulness in ED has not been studied.

Methods: NT-proBNP levels obtained from patients who were suspected as KD at present in ED, from January 1, 2011 to December 31, 2013. The patients were divided into KD with less than 5 febrile days and other febrile disease group according to final diagnosis. The difference of each group, thesensitivity and specificity of NT-proBNP value were analyzed.

Results: In the group of less than 5 febrile days, NT-proBNP levels were significantly higher in the KD patients ( $P=0.01)$. In the receiver operating characteristic curve analysis in the group of less than 5 febrile days, the sensitivity was $62.5 \%$, and the specificity was $77.7 \%$ at $105.5 \mathrm{pg} / \mathrm{mL}$ of NT-proBNP level.

Conclusion: We expect that NT-proBNP would be a useful adjuvant diagnostic tool for KD patients with atypical clinical presentations, especially in cases with less than 5 febrile days.

Key Words: Kawasaki disease; Pro-brain natriuretic peptide

\section{Corresponding Author Hyuksool Kwon}

Department of Emergency Medicine, Seoul National University Bundang Hospital 82, Gumi-ro 173 Beon-gil Bundang-gu, Seongnam-si, Gyeonggi-do 463-707, Korea

Tel: +82-31-787-6351 Fax: +82-31-787-4095

E-mail: jinuking3ganaver.com

\section{Introduction}

Kawasaki disease (KD) is an acute systemic vasculitic syndrome most common in children ${ }^{1)}$. The diagnosis of $\mathrm{KD}$ is based mainly on clinical feature 
(Table 1) and no specific diagnostic test exists. Several studies reported N-terminal pro-brain natriuretic peptide (NT-proBNP) level in patients with the acute phase of $\mathrm{KD}$ as a useful marker of diagnosis ${ }^{2-4)}$.

Determining of KD in emergency department (ED) is diagnostic challenge if the duration of febrile days is not clear. The delayed diagnosis of $\mathrm{KD}$ in the ED might cause confirmative diagnosis of KD about three, four, or more days, and it can increase the risk of coronary artery aneurysm.

Many studies showed NT-proBNP may help diagnosis $\mathrm{KD}^{2-4}$. But, the appropriate level of NT-proBNP for differentiating KD in ED has not yet been suggested. The purpose of this study was to determine whether the serum NT-proBNP level could be a useful marker for patients with the acute phase of $\mathrm{KD}$ in $\mathrm{ED}$, especially when febrile duration was less than 5days. And we also planned to evaluate the performance of NTproBNP in diagnosing $\mathrm{KD}$ to estimate the threshold for differentiating KD from other febrile illnesses in ED.

\section{Methods}

From January 2011 to December 2012, children aged 1 month to 10 years who presented at ED with symptoms suspected KD with less than five febrile days. They underwent NT-proBNP sampling in ED.

And KD group was defined following classical diagnostic criteria as shown in Table 1. Demographic

Table 1. Diagnostic criteria for Kawasaki disease.

Fever lasting for at least five days
At least four of the five following features
Bilateral bulbar conjunctival injection
Changes in the mucosa of the oropharynx
Changes of the peripheral extremities
Polymorphous rash
Cervical adenopathy ( $\geq 1.5 \mathrm{~cm}$ diameter)
Illness not explained by other known disease processes

Pediatric Emergency Medicine Journal data, such as the age or sex, and clinical data, such as the day of illness were compared between KD and controls. Patients with less than 5 febrile days were grouped separately in both groups. Patients with uncorrected significant structural heart disease, known cardiomyopathy, septicemia or septic shock, hypovolemic shock, prolonged fever of unknown etiology, renal failure, patients undergoing or who had undergone chemotherapy involving cardiotoxic drugs and a history of confirmed KD were excluded. NT-proBNP levels of KD with less than five days of fever were compared and Receiver Operator Characteristic (ROC) analysis were obtained for determining cutoff value.

Statistical analyses were performed with STATA 13.1 software (Stata Corp LP). Data are expressed either as median; interquartile range for continuous data or median (range) for categorical data. Nonparametric analysis (Mann-Whitney U test) was utilized for continuous data that did not follow a normal distribution. Rank sum test was also used to compare paired data and to compare grades. ROC analysis was performed to define the serum NT-proBNP concentration at which the sensitivity and specificity were optimal. Odds ratios and $95 \%$ confidence intervals $(95 \%$ Confidence Interval, CI) were calculated. Fisher exact test and chi-square test with Yates correction for continuity were used for categorical data distribution.

\section{Results}

One hundred and eighty one patients were included. Twenty two patients were excluded, 29 patients were eligible for the KD group and 130 patients with other febrile illnesses were enrolled as controls finally (Fig. 1). Sixteen patients in the KD group and 94 patients in the other febrile illness group had less than 4 febrile days when presented at ED. Patients diagnosed with $\mathrm{KD}$ at $\mathrm{ED}$ or outpatient clinic during the 
period of follow-up after discharge from ED fulfilled the Criteria for Diagnostic Guideline established by the Kawasaki Disease Research Committee in Japan. Serum NT-proBNP level was measured at least once in all patients at the time of admission to ED.

There was no significant difference in the age, sex and duration of fever between KD and other febrile illness groups (Table 2). There was a higher serum NT-proBNP level in KD group (322.8 [14.9$1579.3]$ vs 80.0 [6.7-488.9] $\mathrm{pg} / \mathrm{mL} ; \mathrm{P}=0.01)$ in comparison with the patients in the other febrile disease group (Table 2).

The serum NT-proBNP levels to KD in patients with less than 5 febrile days were assessed by a receiver operating characteristic curves analysis
(Fig. 2). A NT-proBNP cut-off value of 105.5 $\mathrm{pg} / \mathrm{mL}$ yielded a sensitivity of $62.5 \%$, a specificity of $73.0 \%$. The area under the curve of NTproBNP for predicting KD in less than 5 febrile group was 0.701 (Fig. 2).

\section{Discussion}

The results of this study show that serum NTproBNP may be useful as a clinical marker for diagnosis of acute phase of $\mathrm{KD}$ in $\mathrm{ED}$ in the case of less than 5 febrile days. The cut-off value of NT-proBNP is $105.5 \mathrm{pg} / \mathrm{mL}$ and sensitivity and specificity is $62.5 \%$ and $73.0 \%$.

Fever, bilateral non-exudative conjunctivitis,

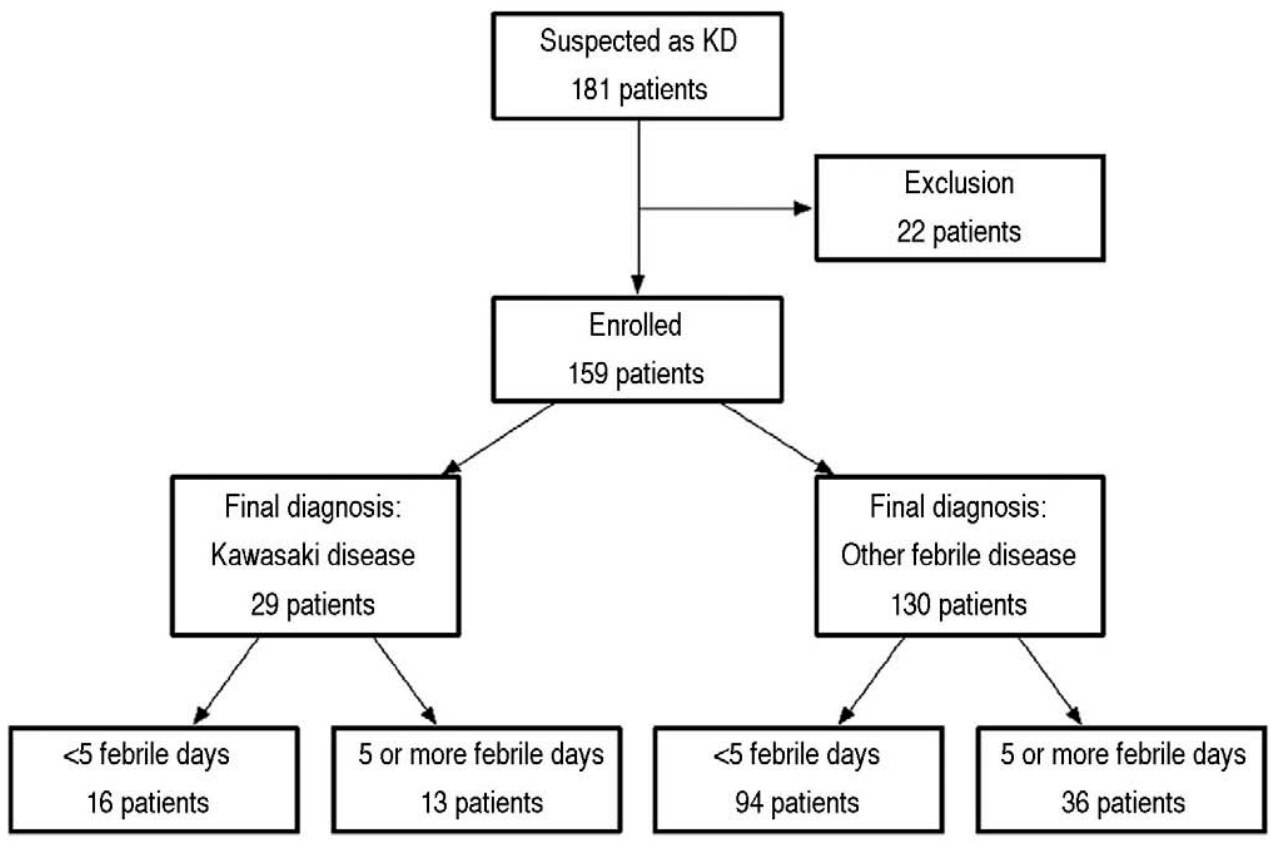

Fig. 1. Patients enrollment.

KD: Kawasaki disease

Table 2. Demographic characteristics in KD and other febrile illness, in the cases of less than 5 days of fever.

\begin{tabular}{lccc}
\hline & KD & Other febrile illness & P value \\
\hline Age, month (median, IQR) & $47.5(5-117)$ & $49.2(8-129)$ & 0.841 \\
Male sex (\%) & 65.5 & 53.8 & 0.304 \\
Duration of fever, day (median IQR) & $3.0(2.0-4.0)$ & $3.0(2.0-4.0)$ & 0.501 \\
NT-proBNP (pg/mL), median (IQR) & $322.8(14.9-1579.3)$ & $80.0(6.7-488.9)$ & 0.009 \\
\hline
\end{tabular}

KD: Kawasaki disease, IQR: interquartile range, NT-pro-BNP: N-terminal pro-brain natriuretic peptide 
erythema of the lips and oral mucosa, changes in the extremities, polymorphous exanthem, and cervical lymphadenopathy are classical clinical presentation of $\mathrm{KD}^{1,5)}$. But, these indicators are

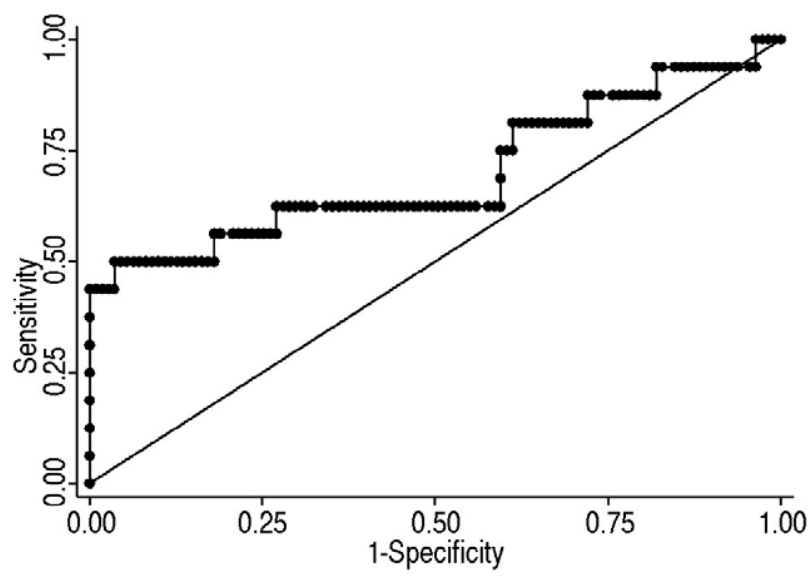

Fig. 2. Receiver operating characteristic curve. Area under the curve of NT-proBNP for predicting Kawasaki disease in less than 5 febrile days; 0.7005. NT-proBNP: N-terminal pro-brain natriuretic peptide. not all present on any given day in many cases ${ }^{6)}$. Some laboratory findings can help in identifying KD: the presence of raised white blood cell count white blood cells in microscopy of urine analysis, and raised transaminases or bilirubin ${ }^{1,6,7)}$. However, these tests are neither pathognomonic nor diagnostic. Diagnosis and treatment are delayed to confirm a fever persisting for at least 5 days in patients presenting with incomplete clinical features of $\mathrm{KD}^{2)}$.

There is no report of diagnosis of $\mathrm{KD}$ in $\mathrm{ED}$. It seems that diagnosis of KD is more difficult than in clinic especially when duration of fever is less than 5 days or the symptoms are incomplete. KD is associated with the elevation of inflammatory markers including Erythrocyte Sedimentation Ratio (ESR), C-Reactive Protein (CRP) and platelet count ${ }^{8)}$. Using the algorithm of the American Heart Association (AHA) for diagnosis of incomplete $\mathrm{KD}$, laboratory tests are recommended when a patient has fever in addition to two or more clinical criteria

Table 3. Supplemental laboratory criteria (A) and echocardiographic criteria (B) for the diagnosis of incomplete Kawasaki disease prepared by the American Heart Association.

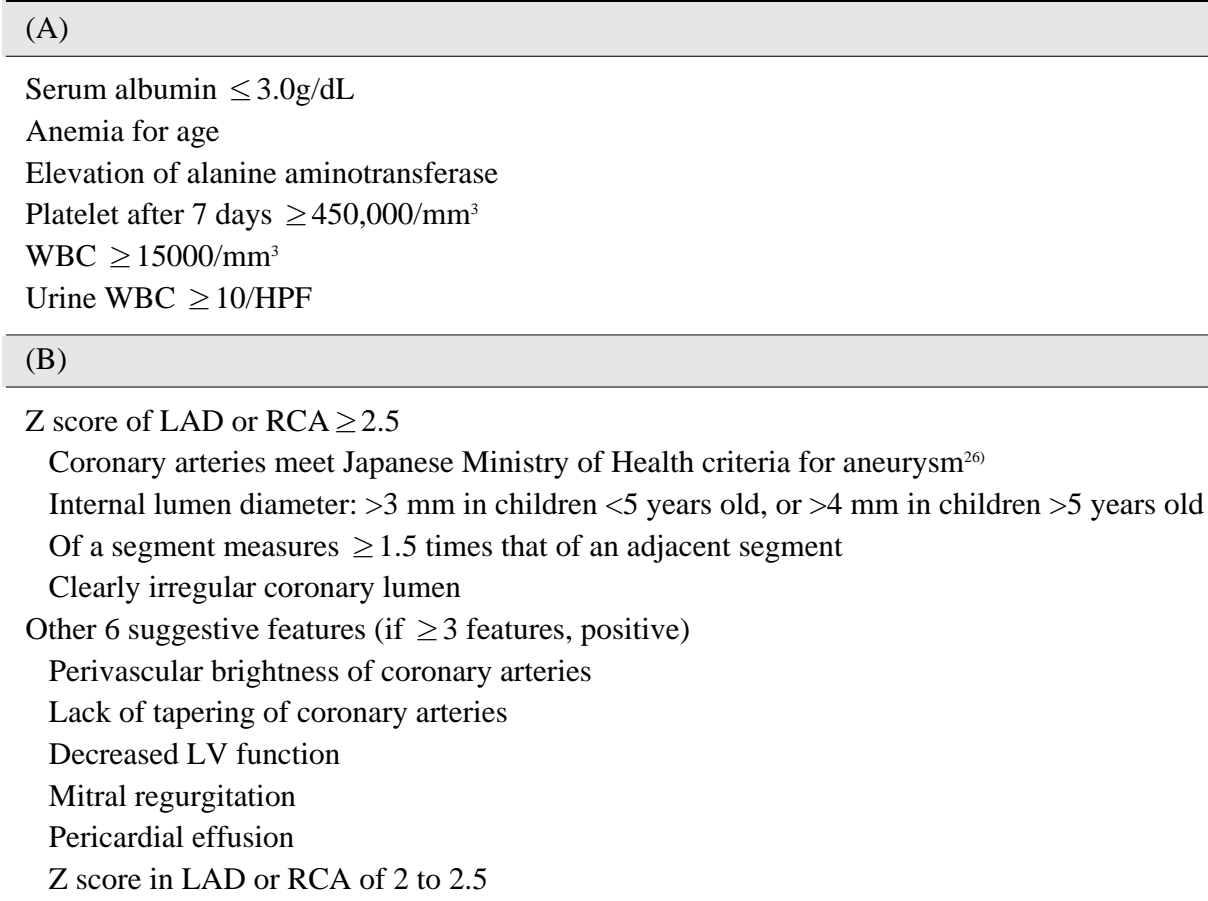

WBC: white blood cell count in CBC (complete blood cell count), HPF: high power field, LAD: left anterior descending coronary artery, RCA: right coronary artery, LV: left ventricle. 
of $\mathrm{KD}^{9}$. Incomplete Kawasaki disease should be considered in all children with unexplained fever for $\geq 5$ days associated with 2 or 3 of the principal clinical features of Kawasaki disease. The AHA recommended a diagnostic algorithm of incomplete Kawasaki disease which comprises of 6 supplemental laboratory and echocardiographic criteria (Table 3). Echocardiography and intravenous immunoglobulin administration should be considered when the CRP level is $3 \mathrm{mg} / \mathrm{dL}$ or higher or when the ESR is 40 $\mathrm{mm} / \mathrm{h}$ or faster. But, these laboratory tests can be increased in other febrile diseases in ED and these result can be useless in differentiation of $\mathrm{KD}$ from other illness in ED frequently.

Several studies reported increased plasma NTproBNP level in patients with the acute phase of KD thus providing a useful marker for diagnosing $\mathrm{KD}^{2-4,10,11}$. Dahdah et al. reported pre-intravenous immunoglobulin NT-proBNP levels were significantly higher in KD patients than in controls (923.6 \pm 1361.7 vs. $186.2 \pm 198.0 \mathrm{ng} / \mathrm{L} ; \mathrm{P}\langle 0.001)^{2)}$. Cho et al. ${ }^{3)}$ reported NT-proBNP levels were significantly higher in the KD patients than in the controls (P 40.001$)$. In the ROC curve analysis, the sensitivity was $66.1 \%$, and the specificity was $77.1 \%$ at $235.2 \mathrm{pg} / \mathrm{mL}$. NT-proBNP is the best available predictive value according to these studies, unlike the currently used laboratory tests. But there had been no evaluated study about usefulness of NTproBNP level in ED as an adjuvant laboratory

\section{REFERENCES}

1. Wood LE, Tulloh RMR. Kawasaki disease in children. Heart 2009;95:787-92.

2. Dahdah N, Siles A, Fournier A, Cousineau J, Delvin E, Saint-Cyr C, et al. Natriuretic peptide as an adjunctive diagnostic test in the acute phase of Kawasaki disease. Pediatr Cardiol 2009;30:810-7.

3. Cho SY, Kim Y, Cha SH, Suh JT, Han MY, Lee HJ. Adjuvant laboratory marker of Kawasaki disease; NTproBNP or hs-CRP? Ann Clin Lab Sci 2011;41:360-3.

4. McNeal-Davidson A, Fournier A, Spigelblatt L, Saint-Cyr C, Mir TS, Nir A, et al. Value of amino-terminal pro Bnatriuretic peptide in diagnosing Kawasaki disease. Pediatr Int 2012;54:627-33. marker of acute phase of KD. A NT-proBNP cutoff value of $105.5 \mathrm{pg} / \mathrm{mL}$ yielded a sensitivity of $62.5 \%$, a specificity of $73.0 \%$ in this study. Shorter febrile days of this study population might affect the lower value of NT-proBNP compared with other studies.

This study has several limitations. First, this study includes its retrospective nature and the numbers of patients are different in $\mathrm{KD}$ and control group. Second, the statistical conclusion might be influenced by the small number of patients with KD and non-parametric statistical tests performed over. Further study will be required for comparison between similar groups. Third limitation may have been related to the variety in the diagnoses of the febrile controls. The categories of febrile diseases should be considered future study.

\section{Conclusion}

These findings will doubtless be much scrutinized, but there is a immediately dependable conclusion that NT-proBNP can be an adjuvant laboratory marker for $\mathrm{KD}$ in $\mathrm{ED}$ if the fever duration is less than 5 days. A prospective multicenter study with emphasis on NT-proBNP in a diagnostic algorithm of $\mathrm{KD}$ in $\mathrm{ED}$ is needed.

5. Dajani AS, Taubert KA, Gerber MA, Shulman ST, Ferrieri P, Freed M, et al. Diagnosis and therapy of Kawasaki disease in children. Circulation 1993;87:1776-80.

6. Kim DS. Kawasaki disease. Yonsei Med J 2006;47:759-72.

7. Sanchez-Manubens J, Bou R, Anton J. Diagnosis and classification of Kawasaki disease. J Autoimmun 2014; 48-49:113-7.

8. Nigrovic LE, Nigrovic PA, Harper MB, Chiang VW. Extreme thrombocytosis predicts Kawasaki disease in infants. Clin Pediatr (Phila) 2006;45:446-52.

9. Newburger JW, Takahashi M, Gerber MA, Gewitz MH, Tani LY, Burns JC, et al. Diagnosis, treatment, and longterm management of Kawasaki disease: a statement for 
health professionals from the Committee on Rheumatic Fever, Endocarditis, and Kawasaki Disease, Council on Cardiovascular Disease in the Young, American Heart Association. Pediatrics 2004;114:1708-33.

10. No SJ, Kim DO, Choi KM, Eun LY. Do predictors of incomplete Kawasaki disease exist for infants? Pediatr Cardiol 2013;34:286-90.

11. Iwashima S, Ishikawa T. B-type natriuretic peptide and Nterminal proBNP in the acute phase of Kawasaki disease. World J Pediatr 2013;9:239-44. 